\title{
Investigating the Role of Environmental Transmission in COVID-19 Dynamics: A Mathematical Model Based Study
}

Ibrahim M. ELmojtaba

Sultan Qaboos University

Fatma Al-Musalhi

Sultan Qaboos University

Asma Al-Ghassani

Sultan Qaboos University

Nasser Al-Salti ( $\square$ nalsalti@squ.edu.om )

Sultan Qaboos University https://orcid.org/0000-0001-9726-4624

\section{Research Article}

Keywords: COVID-19, Environmental Transmission, Basic Reproduction Number, Stability Analysis, Sensitivity Analysis

Posted Date: June 2nd, 2020

DOl: https://doi.org/10.21203/rs.3.rs-32476/v1

License: (9) This work is licensed under a Creative Commons Attribution 4.0 International License. Read Full License 


\title{
Investigating the Role of Environmental Transmission in COVID-19 Dynamics: A Mathematical Model Based Study
}

\author{
Ibrahim M. ELmojtaba, Fatma Al-Musalhi, Asma Al-Ghassani and Nasser Al-Salti \\ Sultan Qaboos University, 123 AL-Khoud, Oman
}

\begin{abstract}
A mathematical model with environmental transmission has been proposed and analyzed to investigate its role in the transmission dynamics of the ongoing COVID-19 outbreak. Two expressions for the basic reproduction number $R_{0}$ have been analytically derived using the next generation matrix method. The two expressions composed of a combination of two terms related to human to human and environment to human transmissions. The value of $R_{0}$ has been calculated using estimated parameters corresponding to two datasets. Sensitivity analysis of the reproduction number to the corresponding model parameters has been carried out. Existence and stability analysis of disease free and endemic equilibrium points have been presented in relation with the obtained expressions of $R_{0}$. Numerical simulations to demonstrate the effect of some model parameters related to environmental transmission on the disease transmission dynamics have been carried out and the results have been demonstrated graphically.
\end{abstract}

Keywords: COVID-19, Environmental Transmission, Basic Reproduction Number, Stability Analysis, Sensitivity Analysis.

\section{Introduction}

The ongoing novel coronavirus disease (COVID-19) outbreak, emerged in Wuhan, China in December 2019, has been declared a pandemic by the World Health Organization (WHO) on March 11, 2020 [1]. It has spread in over 210 countries and territories around the world with more than 3.5 millions confirmed infections and around 250 thousands reported death cases worldwide as of May 4, 2020.

Coronavirus is a family of pathogens that primarily targets the human respiratory system. Previous outbreaks of coronaviruses $(\mathrm{CoVs})$ include the severe acute respiratory syndrome 
SARS-CoV and the Middle East respiratory syndrome MERS-CoV which have been previously characterized as agents that pose a great public health threat [20]. The new strain of this family, causing COVID-19, has been named SARS-CoV2 by WHO on February, 2020 [2]. In the early stage of COVID-19 outbreak, most of the confirmed cases were found to have a link with the Huanan Seafood Wholesale Market, which was closed on January 1, 2020 [3]. COVID-19 can be transmitted directly from person to person by respiratory droplets, and emerging evidence suggested that it may also be transmitted through contact and fomites [13]. Previous studies suggested that the transmission of coronaviruses might occur from contaminated dry surfaces [10, 18], and recently Kampf et. al. concluded that coronaviruses can remain infectious on inanimate surfaces for up to 9 days [14]. Moreover, Ong et. al [17] concluded their study by stating that significant environmental contamination by patients with SARS-CoV2 through respiratory droplets and fecal shedding suggests the environment as a potential medium of transmission and supports the need for strict adherence to environmental and hand hygiene. Therefore it is very essential to investigate this route of transmission in the global spread of COVID-19. Hence, the aim of this work is to study the role of environmental contamination by infected individuals in COVID-19 transmission dynamics through mathematical modeling. A number of mathematical models have been already developed to study the transmission dynamics of COVID-19, see for example [7, 15, 16, 22, 23, 26]. In [7], the authors developed a Bat-Host-Reservoir-People model and then reduced it to a Reservoir-People model in order to focus in the transmission from Huanan Seafood Wholesale Market (reservoir) to people. They have estimated the value of $R_{0}$ from reservoir to human to be 2.3 and from human to human to be 3.58. In general, the most commonly used model is the Susceptible- ExposedInfectious-Recovered model (SEIR) with the infectious individuals taken as one class as in [26] or divided into symptomatic and asymptomatic classes as in [22]. In this work, we will follow the same approach, but we include a new route of disease transmission corresponding to human contact with contaminated environment. The rest of the paper is organized as follows. In the next section, we present the proposed mathematical model. The mathematical analysis of the proposed model will be carried out in Section 3. The analysis includes the calculation of the basic reproduction number, sensitivity analysis and stability analysis. In Section 4, numerical simulations to demonstrate the effect of some model parameters related to the environmental transmission in the disease transmission dynamics are carried out. Finally, a brief conclusion is presented in Section 5 . 


\section{Model Formulation}

To investigate the role of environmental transmission in the COVID-19 dynamics, we propose the following model:

$$
\begin{aligned}
S^{\prime}(t) & =\Lambda-\beta_{1} I(t) \frac{S(t)}{N(t)}-\beta_{2} A(t) \frac{S(t)}{N(t)}-\frac{\beta_{e} B(t) S(t)}{k+B(t)}-\mu S(t) \\
E^{\prime}(t) & =\beta_{1} I(t) \frac{S(t)}{N(t)}+\beta_{2} A(t) \frac{S(t)}{N(t)}+\frac{\beta_{e} B(t) S(t)}{k+B(t)}-(\lambda+\mu) E(t) \\
A^{\prime}(t) & =\epsilon \lambda E(t)-\left(\mu+\gamma_{2}\right) A(t) \\
I^{\prime}(t) & =(1-\epsilon) \lambda E(t)-\left(\mu+\gamma_{1}+\delta\right) I(t) \\
R^{\prime}(t) & =\gamma_{1} I(t)+\gamma_{2} A(t)-\mu R(t) \\
B^{\prime}(t) & =\alpha_{1} I(t)+\alpha_{2} A(t)-\mu_{e} B(t)
\end{aligned}
$$

where, $N(t)$ is the total human population, which is divided into susceptible $S(t)$, exposed $E(t)$, asymptomatic $A(t)$, infected $I(t)$ and recovered $R(t)$ classes, so that $N(t)=S(t)+E(t)+A(t)+$ $I(t)+R(t)$ and $N^{\prime}(t)=\Lambda-\mu N(t)-\delta I(t)$. Here $B(t)$ represents the concentration of coronavirus at contaminated environment and as it is the case for most models involving environmental transmission, see for example [24, 4] and the references therein, the environmental-related force of infection is modeled using Michealis-Menten or Holling type II functional responses, taking to be $\frac{\beta_{e} B}{k+B}$ in our model, where $\beta_{e}$ is the contact rate with the contaminated environment and $\frac{B}{k+B}$ is the probability of catching the disease. The constant $k$ represents the minimum concentration of virus at environment capable of ensuring $50 \%$ chance of contracting the disease. The minimum infectious dose for human to human transmission of COVID-19 is expected to be very small. However, for infection through environmental transmission, it is expected to be a lot more, since the virus needs to first survive at the surface before starting the journey from surface through hand to face [9]. All other parameters of model (1) are defined in Table 1. 
Table 1: Parameters used in model (1)

\begin{tabular}{|l|l|}
\hline Parameter & Symbol \\
\hline Natural death/birth rate & $\mu$ \\
Disease related death rate of humans & $\delta$ \\
Recruitment Rate & $\Lambda$ \\
Contact rate from contaminated environment & $\beta_{e}$ \\
Shedding rate from symptomatic to environment & $\alpha_{1}$ \\
Shedding rate from asymptomatic to environment & $\alpha_{2}$ \\
Life time of the virus in the environment & $1 / \mu_{e}$ \\
Transmission rate of the disease from symptomatic & $\beta_{1}$ \\
Transmission rate of the disease from asymptomatic & $\beta_{2}$ \\
Rate at which exposed become symptomatic & $\lambda$ \\
Proportion of asymptomatic individuals & $\epsilon$ \\
Recovery rate of symptomatic individuals & $\gamma_{1}$ \\
Recovery rate of asymptomatic individuals & $\gamma_{2}$ \\
\hline
\end{tabular}

\section{Mathematical Analysis of the Proposed Model}

\subsection{Normalized Model}

Model (1) can be rewritten using the following set of normalized variables:

$$
\begin{aligned}
& \tilde{N}=\frac{N}{\mathcal{N}}, \quad \tilde{S}=\frac{S}{\mathcal{N}}, \quad \tilde{E}=\frac{E}{\mathcal{N}}, \quad \tilde{A}=\frac{A}{\mathcal{N}}, \\
& \tilde{I}=\frac{I}{\mathcal{N}}, \quad \tilde{R}=\frac{R}{\mathcal{N}}, \quad \tilde{B}=\frac{B}{\mathcal{B}},
\end{aligned}
$$


where $\mathcal{N}=\Lambda / \mu$ and $\mathcal{B}=\left(\alpha_{1} \Lambda\right) /\left(\mu_{e} \mu\right)$. We then obtain the following normalized model:

$$
\begin{aligned}
S^{\prime}(t) & =\mu-\beta_{1} I(t) \frac{S(t)}{N(t)}-\beta_{2} A(t) \frac{S(t)}{N(t)}-\frac{\beta_{e} B(t) S(t)}{K+B(t)}-\mu S(t) \\
E^{\prime}(t) & =\beta_{1} I(t) \frac{S(t)}{N(t)}+\beta_{2} A(t) \frac{S(t)}{N(t)}+\frac{\beta_{e} B(t) S(t)}{K+B(t)}-(\lambda+\mu) E(t) \\
A^{\prime}(t) & =\epsilon \lambda E(t)-\left(\mu+\gamma_{2}\right) A(t) \\
I^{\prime}(t) & =(1-\epsilon) \lambda E(t)-\left(\mu+\gamma_{1}+\delta\right) I(t) \\
R^{\prime}(t) & =\gamma_{1} I(t)+\gamma_{2} A(t)-\mu R(t) \\
B^{\prime}(t) & =\mu_{e} I(t)+\alpha \mu_{e} A(t)-\mu_{e} B(t)
\end{aligned}
$$

\begin{tabular}{|c|c|c|c|c|}
\hline Parameter & \multicolumn{4}{|c|}{ Value [ref.] } \\
\hline$\mu$ & \multicolumn{2}{|c|}{$0.3589 \times 10^{-4}$} & \multicolumn{2}{|l|}{22} \\
\hline$\delta$ & & \multicolumn{2}{|l|}{22} \\
\hline $1 / \mu_{e}$ & \multicolumn{2}{|l|}{5} & \multicolumn{2}{|l|}{14} \\
\hline$\beta_{e}$ & \multicolumn{2}{|l|}{0.5} & \multicolumn{2}{|l|}{ assumed } \\
\hline$\alpha$ & \multicolumn{2}{|l|}{0.5} & \multicolumn{2}{|l|}{ assumed } \\
\hline$K$ & \multicolumn{2}{|l|}{0.7} & \multicolumn{2}{|l|}{ assumed } \\
\hline$\beta_{1}$ & 0.84 & [7] & 1.8457 & 22 \\
\hline$\beta_{2}=c \beta_{1}$ & $c=0.5$ & [7] & $c=0.45$ & 22 \\
\hline$\lambda$ & $1 / 5.2$ & 7] & $1 / 7$ & 22 \\
\hline$\epsilon$ & 0.5 & 7] & 0.13166 & 22 \\
\hline$\gamma_{1}$ & 0.1724 & 7 & 0.46 & 22 \\
\hline$\gamma_{2}$ & 0.1724 & [7] & 0.2561 & 22 \\
\hline
\end{tabular}

where all over tildes have been neglected for ease of notation. Here $\alpha=\left(\alpha_{2} / \alpha_{1}\right)$ and $K=$ $\frac{k}{\left(\alpha_{1} \Lambda\right) /\left(\mu_{e} \mu\right)}$ represent the relative shedding rate of asymptomatic humans and the relative minimum concentration of virus at environment capable of ensuring $50 \%$ chance of contracting the disease, respectively. The values of the parameters used in model (2) are listed in Table 2.

Table 2: Values of the parameters used in model (2) 


\subsection{The Basic Reproduction Number}

We first start by calculating the basic reproduction number using the next generation matrix method [8]. For this purpose, we need to find the disease free equilibrium (DFE) of the model, the matrix of transmission terms (the production of new infections) and the matrix of transition terms (the change of state terms). The DFE of the model is $E_{0}=(1,0,0,0,0,0)$, the matrix of transmission terms is

$$
\mathcal{F}=\left[\begin{array}{c}
\beta_{1} I \frac{S}{N}+\beta_{2} A \frac{S}{N}+\frac{\beta_{e} B S}{K+B} \\
0 \\
0 \\
0
\end{array}\right]
$$

and the matrix of transition terms is

$$
\mathcal{V}=\left[\begin{array}{c}
(\mu+\lambda) E \\
-\epsilon \lambda E+\eta A \\
-(1-\epsilon) \lambda E-\xi I \\
-\mu_{e} I-\alpha \mu_{e} A+\mu_{e} B
\end{array}\right]
$$

where $\xi=\left(\mu+\gamma_{1}+\delta\right)$ and $\eta=\left(\mu+\gamma_{2}\right)$. Calculating the Jacobian of these two matrices at the DFE, we get

$F=$ Jacobian of $\mathcal{F}$ at $E_{0}=\left[\begin{array}{cccc}0 & \beta_{2} & \beta_{1} & \frac{\beta_{e}}{K} \\ 0 & 0 & 0 & 0 \\ 0 & 0 & 0 & 0 \\ 0 & 0 & 0 & 0\end{array}\right]$

and

$V=$ Jacobian of $\mathcal{V}$ at $E_{0}=\left[\begin{array}{cccc}\mu+\lambda & 0 & 0 & 0 \\ -\epsilon \lambda & \eta & 0 & 0 \\ -(1-\epsilon) \lambda & 0 & \xi & 0 \\ 0 & -\alpha \mu_{e} & -\mu_{e} & \mu_{e}\end{array}\right]$.

Hence, the next generation matrix is

$$
F V^{-1}=\left[\begin{array}{ccccc}
\frac{\beta_{2} \epsilon \lambda}{\eta(\lambda+\mu)}+\frac{\beta_{1} \lambda(1-\epsilon)}{(\lambda+\mu) \xi}+\frac{\beta_{e} \lambda(\alpha \epsilon \xi+(1-\epsilon) \eta)}{K(\lambda+\mu) \eta \xi} & \frac{\beta_{2}}{\eta}+\frac{\beta_{e} \alpha}{K \eta} & \frac{\beta_{1}}{\xi}+\frac{\beta_{e}}{K \xi} & \frac{\beta_{e}}{\mu_{e} K} \\
0 & 0 & 0 & 0 \\
0 & 0 & 0 & 0 \\
0 & 0 & 0 & 0
\end{array}\right] .
$$


Thus, we have the following expression for the basic reproduction number:

$$
R_{0}=R_{h h}+R_{h e}
$$

where

$$
R_{h h}=\frac{\beta_{2} \epsilon \lambda}{(\lambda+\mu) \eta}+\frac{\beta_{1} \lambda(1-\epsilon)}{(\lambda+\mu) \xi}
$$

and

$$
R_{h e}=\frac{\beta_{e} \alpha \epsilon \lambda}{K(\lambda+\mu) \eta}+\frac{\beta_{e} \lambda(1-\epsilon)}{K(\lambda+\mu) \xi}
$$

The expressions $R_{h h}$ and $R_{h e}$ denote the parts of $R_{0}$ corresponding to human to human and environment to human transmissions, respectively. Based on the parameter values listed in Table 2, we have the following estimated values for $R_{h h}$ and $R_{h e}$ :

Table 3: Estimated Values of $R_{h h}$ and $R_{h e}$

\begin{tabular}{|l|cc|}
\hline Reference & Chen et. al [7] & Nadim et.al [22] \\
\hline$R_{h h}$ & 3.25 & 3.67 \\
$R_{h e}$ & 2.77 & 1.44 \\
\hline
\end{tabular}

Note that each value of $R_{h h}$ is very close to the one obtained in the corresponding reference of the used parameter values. The difference is due to the value of the disease related death rate $\delta$ which was taken to be zero in both references. Moreover, $\mu$ was taken to 0.0018 in [7]. If the values of these two parameters are taken to be the same as the ones in these two references, then the value of $R_{h h}$ will be matching with the corresponding reference, namely, $R_{h h}=3.58$ [7] and $R_{h h}=3.9098$ [22]. The main point we want do address here is the difference between the two values of $R_{h e}$. The value of $R_{h e}$ using parameter values from [7] is almost double the one obtained using parameter values from [22]. We think that the main reason is due to the time frame of the dataset used in each reference. In [7], the dataset was from December 7, 2019 to January 1, 2020 and it was from January 22 to February 21, 2020 in [22] and since the Huanan Seafood Wholesale Market was closed on January 1, 2020, one would expect that the environmental transmission would be less after the closure of the market. Moreover, comparing the values of $R_{h h}$ and $R_{h e}$, we note that in general the contribution of environmental transmission is less compared to human to human transmission.

Remark 3.1. Note that if we consider the term $\alpha_{1} I(t)+\alpha_{2} A(t)$ to be a new infection term (i.e. to be part of the vector $\mathcal{F}$ ), see for example [4, 25, 11, 21], then the basic reproduction 
number will be given by

$$
\tilde{R}_{0}=\frac{1}{2}\left(R_{h h}+\sqrt{R_{h h}^{2}+4 R_{h e}}\right) .
$$

Note that this basic reproduction number has a term with a square root, which indicates that the transmission from human to human via environment takes place in two steps [27]. For this reason, we believe that this expression gives a better representation for the basic reproduction number. Moreover, we should note here that the two forms of the basic reproduction number have the same threshold, i.e., $R_{0}=\tilde{R}_{0}=1$ whenever $R_{h h}+R_{h e}=1$ and hence $R_{0}, \tilde{R}_{0} \lessgtr 1$ whenever $R_{h h}+R_{h e} \lessgtr 1$.

Using the parameter values in Table 2 , the estimated values of $R_{0}$ and $\tilde{R}_{0}$ are given in the following table:

Table 4: Estimated Values of $R_{0}$ and $\tilde{R}_{0}$

\begin{tabular}{|l|cc|}
\hline Reference & Chen et. al [7] & Nadim et.al [22] \\
\hline$R_{0}$ & 6.02 & 5.11 \\
\hline$\tilde{R}_{0}$ & 3.95 & 4.03 \\
\hline
\end{tabular}

The contribution of the environmental transmission in $R_{0}$ is clear, since it increases its value by $R_{h e}$. However, the values of $\tilde{R}_{0}$ are just slightly higher than the corresponding ones without environmental transmission listed in Table 3, indicating that the major contribution is coming from human to human transmission. In general, the obtained values of $R_{0}$ and $\tilde{R}_{0}$ are still within the range of the previously obtained values of the basic reproduction number, see for example [19, 28, 29]. In the following section, we will perform sensitivity analysis to identify the most influential parameters that have significant impact on the basic reproduction number and should be targeted by control measures and intervention strategies.

\subsection{Sensitivity analysis}

Here we adopt the normalized forward sensitivity index, which is also known as elasticity analysis, to study the sensitivity of the basic reproduction number to various model parameters and hence identify the parameters that have high impact on the transmission of the disease. The elasticity index, $\Upsilon_{\phi}^{R_{0}}$, is defined as the relative change of $R_{0}$ to the relative change in the parameter $\phi[12]$, i.e,

$$
\Upsilon_{\phi}^{R_{0}}=\frac{\partial R_{0}}{\partial \phi} \frac{\phi}{R_{0}} .
$$


Using the obtained explicit expressions of the basic reproduction number, one can easily obtain analytic expression of elasticity index with respect to each model parameter. The estimated values of the elasticity indices are obtained using the parameter values taken from [22] as listed in Table 2, The obtained results are listed in Table 5.

Table 5: Sensitivity analysis of model $(2)$

\begin{tabular}{|l|c|c|c|c|}
\hline Parameter $(\phi)$ & $\Upsilon_{\phi}^{R_{h h}}$ & $\Upsilon_{\phi}^{R_{h e}}$ & $\Upsilon_{\phi}^{R_{0}}$ & $\Upsilon_{\phi}^{R_{0}}$ \\
\hline$\beta_{1}$ & 0.8837 & - & 0.6348 & 0.7397 \\
$\beta_{2}$ & 0.1163 & - & 0.0835 & 0.0973 \\
$\lambda$ & 0.00025 & 0.00025 & 0.00025 & 0.00023 \\
$\mu$ & -0.00033 & -0.00033 & -0.00033 & -0.00030 \\
$\delta$ & -0.0608 & -0.0600 & -0.0606 & -0.0558 \\
$\epsilon$ & -0.0177 & -0.0047 & -0.0140 & -0.0152 \\
$\gamma_{1}$ & -0.8228 & -0.8123 & -0.8199 & -0.7549 \\
$\gamma_{2}$ & -0.1163 & -0.1276 & -0.1195 & -0.1077 \\
$K$ & - & -1.0000 & -0.2816 & -0.0815 \\
$\alpha$ & - & 0.1276 & 0.0359 & 0.0104 \\
$\beta_{e}$ & - & 1.0000 & 0.2816 & 0.0815 \\
\hline
\end{tabular}

Note that the sign of the elasticity index determines whether the basic reproduction number increases (positive sign) or decreases (negative sign) with the corresponding parameter and the magnitude measures the relative significant of the parameter. The obtained results show that the transmission rate of COVID-19 from symptomatic humans has the maximum positive index and the recovery rate of symptomatic humans has the maximum negative index with both $R_{0}$ and $\tilde{R}_{0}$. Hence, they are the most influential parameters in the disease transmission dynamics. The transmission rate can be reduced by adopting social distancing, maintaining personal hygiene and practicing healthy habits in general. On the other hand, the recovery rate can be increased by following healthy dietary habits, getting enough sleep, reducing stress and carrying out other activities which enhance the immune system. Moreover, the obtained results suggest that the parameters related to environmental transmission, $K, \alpha, \beta_{e}$, may play less of a role in the diseases transmission.

\subsection{Local and global stability of DFE}

This section is dedicated to the stability analysis of the DFE. The result on local stability of the DFE of model (2) is given in the following theorem: 
Theorem 3.1. The DFE of model (2) is locally asymptotically stable if $R_{0}<1\left(\tilde{R}_{0}<1\right)$, otherwise it is unstable.

Proof. First, let us denote the following:

$$
\begin{array}{ll}
R_{h h 1}=\frac{\beta_{2} \epsilon \lambda}{(\lambda+\mu) \eta}, & R_{h h 2}=\frac{\beta_{1} \lambda(1-\epsilon)}{(\lambda+\mu) \xi} \\
R_{h e 1}=\frac{\beta_{e} \alpha \epsilon \lambda}{K(\lambda+\mu) \eta}, & R_{h e 2}=\frac{\beta_{e} \lambda(1-\epsilon)}{K(\lambda+\mu) \xi} .
\end{array}
$$

The local stability of the DFE can be determined by first computing the Jacobian matrix of system (2) at $E_{0}$ as follows:

$$
J_{D F E}=\left[\begin{array}{cccrcc}
-\mu & 0 & -\beta_{2} & -\beta_{1} & 0 & -\frac{\beta_{e}}{K} \\
0 & -(\lambda+\mu) & \beta_{2} & \beta_{1} & 0 & \frac{\beta_{e}}{K} \\
0 & \epsilon \lambda & -\eta & 0 & 0 & 0 \\
0 & (1-\epsilon) \lambda & 0 & -\xi & 0 & 0 \\
0 & 0 & \gamma_{2} & \gamma_{1} & -\mu & 0 \\
0 & 0 & \alpha \mu_{e} & \mu_{e} & 0 & -\mu_{e}
\end{array}\right]
$$

Clearly, the above matrix has two negative eigenvalues, say $\lambda_{1,2}=-\mu$, and the remaining eigenvalues can be calculated from the characteristic equation which is given by

$$
X^{4}+a_{1} X^{3}+a_{2} X^{2}+a_{3} X+a_{4}=0,
$$

where

$$
\begin{aligned}
a_{1}= & \mu_{e}+\xi+\eta+\mu+\lambda, \\
a_{2}= & (\mu+\lambda) \eta\left(1-R_{h h 1}\right)+\xi(\mu+\lambda)\left(1-R_{h h 2}\right)+\mu_{e}(\mu+\lambda+\eta)+\xi\left(\eta+\mu_{e}\right), \\
a_{3}= & (\mu+\lambda) \eta \mu_{e}\left(1-R_{h h 1}-R_{h e 1}\right)+\xi \eta(\mu+\lambda)\left(1-R_{h h}\right)+\xi \mu_{e} \eta \\
& \quad+(\mu+\lambda) \xi \mu_{e}\left(1-R_{h h 2}-R_{h e 2}\right), \\
a_{4}= & -\xi \eta \mu_{e}(\lambda+\mu)\left(R_{h h}+R_{h e}-1\right) .
\end{aligned}
$$

Here, we will use the Routh-Hurtwiz criteria to show that the roots of the above characteristic equation are negative or have negative real parts. Namely, we need to show that the following conditions are satisfied:

- $a_{1}>0, a_{3}>0, a_{4}>0$. Indeed all coefficients of the characteristic equation are positive whenever $R_{h h}+R_{h e}<1$ which implies $R_{0}<1\left(\tilde{R}_{0}<1\right)$ and hence this condition is satisfied. 
- $a_{1} a_{2} a_{3}>a_{3}^{2}+a_{1}^{2} a_{4}$. First, we start by simplifying the term $a_{1} a_{2}-a_{3}$ as follow:

$$
\begin{aligned}
a_{1} a_{2}-a_{3}= & \left(\mu_{e}+\xi+\eta+\lambda+\mu\right)\left[(\mu+\lambda) \eta\left(1-R_{h h 1}\right)+\xi(\mu+\lambda)\left(1-R_{h h 2}\right)\right. \\
& \left.+\mu_{e}(\mu+\lambda+\eta)+\xi\left(\eta+\mu_{e}\right)\right]-\left[(\mu+\lambda) \eta \mu_{e}\left(1-R_{h h 1}-R_{h e 1}\right)+\xi \eta(\mu+\lambda)\left(1-R_{h h}\right)\right. \\
& \left.+(\mu+\lambda) \xi \mu_{e}\left(1-R_{h h 2}-R_{h e 2}\right)+\xi \mu_{e} \eta\right] \\
= & {\left[(\mu+\lambda)\left((\eta+\lambda+\mu) \eta\left(1-R_{h h 1}\right)+(\xi+\eta+\lambda+\mu) \xi\left(1-R_{h h 2}\right)\right)\right.} \\
& \left.+\mu_{e}(\mu+\lambda+\eta)\left(\mu_{e}+\xi+\eta+\lambda+\mu\right)+\xi\left(\eta+\mu_{e}\right)(\xi+\eta+\lambda+\mu)+\xi \mu_{e}^{2}\right] \\
& +\left((\mu+\lambda) \eta \mu_{e}\left(1-R_{h h 1}\right)-(\mu+\lambda) \eta \mu_{e}\left(1-R_{h h 1}-R_{h e 1}\right)\right)+\left((\mu+\lambda) \eta \xi\left(1-R_{h h 1}\right)\right. \\
& \left.-(\mu+\lambda) \eta \xi\left(1-R_{h h}\right)\right)+\left((\mu+\lambda) \xi \mu_{e}\left(1-R_{h h 2}\right)-(\mu+\lambda) \xi \mu_{e}\left(1-R_{h h 2}-R_{h e 2}\right)\right) \\
& +\xi \mu_{e} \eta-\xi \mu_{e} \eta \\
= & (\mu+\lambda)\left((\eta+\lambda+\mu) \eta\left(1-R_{h h 1}\right)+(\xi+\eta+\lambda+\mu) \xi\left(1-R_{h h 2}\right)\right) \\
& +\mu_{e}(\mu+\lambda+\eta)\left(\mu_{e}+\xi+\eta+\lambda+\mu\right)+\xi\left(\eta+\mu_{e}\right)(\xi+\eta+\lambda+\mu)+\xi \mu_{e}^{2} \\
& +(\mu+\lambda)\left(\eta \mu_{e} R_{h e 1}+\eta \xi R_{h h 2}+\xi \mu_{e} R_{h e 2}\right) .
\end{aligned}
$$

Note $a_{1} a_{2}-a_{3}>0$ if $R_{h h 1}<1$ and $R_{h h 2}<1$, which holds true whenever $R_{0}<1\left(\tilde{R}_{0}<1\right)$. Some versions of the Routh-Hurtwiz criteria require $a_{1} a_{2}>a_{3}$ as a separate condition for 
characteristic equations with degree 4 . Then, we have

$$
\begin{aligned}
& a_{1} a_{2} a_{3}-a_{3}^{2}-a_{1}^{2} a_{4}=\left(a_{1} a_{2}-a_{3}\right) a_{3}-a_{1}^{2} a_{4} \\
&=\left((\mu+\lambda)\left((\eta+\lambda+\mu) \eta\left(1-R_{h h 1}\right)+(\xi+\eta+\lambda+\mu) \xi\left(1-R_{h h 2}\right)\right)\right. \\
& \quad+\mu_{e}(\mu+\lambda+\eta)\left(\mu_{e}+\xi+\eta+\lambda+\mu\right)+\xi\left(\eta+\mu_{e}\right)(\xi+\eta+\lambda+\mu) \\
&\left.\quad+\xi \mu_{e}^{2}+(\mu+\lambda)\left(\eta \mu_{e} R_{h e 1}+\eta \xi R_{h h 2}+\xi \mu_{e} R_{h e 2}\right)\right) \times \\
&\left((\mu+\lambda) \eta \mu_{e}\left(1-R_{h h 1}-R_{h e 1}\right)+\xi \eta(\mu+\lambda)\left(1-R_{h h}\right)\right. \\
&\left.+(\mu+\lambda) \xi \mu_{e}\left(1-R_{h h 2}-R_{h e 2}\right)+\xi \mu_{e} \eta\right) \\
&-\left(\mu_{e}+\xi+\eta+\lambda+\mu\right)^{2}(\lambda+\mu) \xi \eta \mu_{e}\left(1-R_{h h}-R_{h e}\right) \\
&=\left(\mu_{e}^{2}+\xi^{2}+\eta^{2}+\lambda^{2}+\mu^{2}+2 \mu_{e} \xi+2 \mu_{e} \eta+2 \mu_{e} \lambda+2 \mu_{e} \mu\right. \\
&+2 \xi \eta+2 \xi \lambda+2 \xi \mu+2 \eta \lambda+2 \eta \mu+2 \lambda \mu) \xi \eta \mu_{e}(\lambda+\mu) \\
&-\left(\mu_{e}+\xi+\eta+\lambda+\mu\right)^{2}(\lambda+\mu) \xi \eta \mu_{e}\left(1-R_{h h}-R_{h e}\right) \\
&+ \text { positive terms } \\
&=\left(\mu_{e}+\xi+\eta+\lambda+\mu\right)^{2}(\lambda+\mu) \xi \eta \mu_{e}\left(R_{h h}+R_{h e}\right)+\text { positive terms }
\end{aligned}
$$

Therefore, the condition $a_{1} a_{2} a_{3}>a_{3}^{2}+a_{1}^{2} a_{4}$ is satisfied and we conclude that the DFE is locally asymptotically stable if $R_{0}<1\left(\tilde{R}_{0}<1\right)$.

Now, we state and prove the global stability result of the DFE of model (2). The result is stated in the following theorem:

Theorem 3.2. The DFE of model (2) is globally asymptotically stable provided that $R_{0}<1$.

Proof. The proof is based on the theorem of Castillo-Chavez and Feng as described in [6]. We first write the model as

$$
\frac{d X}{d t}=F(X, Y)
$$

and

$$
\frac{d Y}{d t}=G(X, Y), G(X, 0)=0
$$

where $X=(S, R)$ and $Y=(E, A, I, B)$, represent uninfected and infected classes, respectively. $F(X, Y)$ and $G(X, Y)$ are the corresponding right hand side of model (2). The DFE of the system can be written as $E_{0}=\left(X_{0}, 0\right)$, where $X_{0}=(1,0)$. To prove global asymptotic stability of DFE, the following conditions must be satisfied: 
(H1) $\frac{d X}{d t}=F(X, 0), X_{0}$ is global asymptotically stable.

(H2) $G(X, Y)=A Y-\widehat{G}(X, Y), \quad \widehat{G}(X, Y) \geq 0$ for $(X, Y) \in \Omega$,

where, $A=D_{Y} G\left(X_{0}, 0\right)$ is an M- matrix and

$\Omega=\left\{(S, E, A, I, R, B) \in \mathbb{R}_{+}^{6} \mid S+E+A+I+R=N \leq 1, B \leq 1\right\}$

is the region where the model makes biological sense. We begin by proving condition (H1), in which we have

$$
\left[\begin{array}{l}
S^{\prime} \\
R^{\prime}
\end{array}\right]=\left[\begin{array}{l}
\mu-\mu S \\
-\mu R
\end{array}\right] .
$$

Solving the above differential equations, we obtain:

$$
S(t)=C_{1} e^{-\mu t}+1, \text { and } R(t)=C_{2} e^{-\mu t},
$$

where $C_{1}$ and $C_{2}$ are constants. Clearly, $X \rightarrow X_{0}$ as $t \rightarrow \infty$. Hence, $X_{0}$ is global asymptotically stable and condition (H1) is satisfied. For condition (H2), we first find the matrix $A$ :

$$
A=\left[\begin{array}{cccc}
-(\lambda+\mu) & \beta_{2} & \beta_{1} & \frac{\beta_{e}}{K} \\
\epsilon \lambda & -\eta & 0 & 0 \\
(1-\epsilon) \lambda & 0 & -\xi & 0 \\
0 & \alpha \mu_{e} & \mu_{e} & -\mu_{e}
\end{array}\right]
$$

and then, we have

$\widehat{G}(X, Y)=A Y-G(X, Y)=\left[\begin{array}{c}\frac{\beta_{2} A}{N}(N-S)+\frac{\beta_{1} I}{N}(N-S)+\frac{\beta_{e} B}{K(K+B)}(K(1-S)+B) \\ 0 \\ 0 \\ 0\end{array}\right]$.

Clearly $\widehat{G}(X, Y) \geq 0$ for all $(X, Y) \in \Omega$. Therefore, the DFE is globally asymptotically stable.

\subsection{Existence and local stability of EE}

In this section, we discuss the existence and local stability of endemic equilibrium (EE). First, let the EE of model (2) be given by

$$
E_{1}=\left(S^{*}, E^{*}, A^{*}, I^{*}, R^{*}, B^{*}\right) .
$$


Then, denote the following:

$$
\Phi=\frac{\beta_{1} I^{*}}{N^{*}}+\frac{\beta_{2} A^{*}}{N^{*}}+\frac{\beta_{e} B^{*}}{K+B^{*}}
$$

where,

$$
N^{*}=\frac{1}{\mu}\left(\mu-\delta I^{*}\right)
$$

Now, the components of the EE, can be written as

$$
\begin{aligned}
& S^{*}=\frac{\mu}{\Phi+\mu}, \quad E^{*}=\frac{\Phi S^{*}}{\lambda+\mu} \\
& A^{*}=\frac{\epsilon \lambda}{\eta} E^{*}, \quad I^{*}=\frac{(1-\epsilon) \lambda}{\xi} E^{*}, \\
& R^{*}=\frac{\gamma_{1} I^{*}+\gamma_{2} A^{*}}{\mu}, \quad B^{*}=C E^{*} .
\end{aligned}
$$

where, $C=\frac{(1-\epsilon) \lambda}{\xi}+\frac{\alpha \epsilon \lambda}{\eta}$. Substituting the above expressions in 4 and simplifying, we obtain the following equation of $\Phi$ :

$$
a_{1} \Phi^{2}+a_{2} \Phi+a_{3}=0
$$

where,

$$
\begin{aligned}
a_{1}= & (K(\lambda+\mu)+C \mu)((\lambda+\mu) \xi-\delta(1-\epsilon) \lambda), \\
a_{2}= & K \mu \xi(\lambda+\mu)^{2}\left(1-R_{h h}-R_{h e}\right)+C \xi \mu^{2}(\lambda+\mu)\left(1-R_{h h}\right)+\delta(1-\epsilon) \lambda \beta_{e} C \mu \\
& +K \mu(\lambda+\mu)((\lambda+\mu) \xi-\delta(1-\epsilon) \lambda), \\
a_{3}= & K \mu^{2} \xi(\lambda+\mu)^{2}\left(1-R_{h h}-R_{h e}\right),
\end{aligned}
$$

One can easily verify that $(\lambda+\mu) \xi-\delta(1-\epsilon) \lambda>0$, which implies that $a_{1}$ is always positive. The existence of EE follows from existence of a positive solution to equation (5), which can be determined as follows:

- If $R_{h h}+R_{h e} \leq 1$, then all coefficients of equation (5) are non-negative and hence there is no positive solution.

- If $R_{h h}+R_{h e}>1$, then $a_{3}<0$ and hence equation (5) has a unique positive solution. 
Therefore, we conclude that a unique EE of model (2) exists if $R_{0}>1\left(\tilde{R}_{0}>1\right)$.

Next, the local stability result of the EE of model (2) is given in the following theorem:

Theorem 3.3. The EE of model (2) is locally asymptotically stable if $R_{0}>1\left(\tilde{R}_{0}>1\right)$.

Proof. The method of proof is based on Theorem 4.1 in Chaves and Song [5], taking $\beta_{e}$ to be a bifurcation parameter. The corresponding bifurcation value at $R_{0}=1\left(\tilde{R}_{0}=1\right)$ is given by

$$
\beta_{e}^{*}=\frac{K\left((\lambda+\mu) \xi \eta-\epsilon \lambda \xi \beta_{2}-(1-\epsilon) \lambda \eta \beta_{1}\right)}{\lambda(\epsilon \xi \alpha+(1-\epsilon) \eta)}
$$

Now at $R_{0}=1$, the Jacobian of system (2) given by (3) clearly has a zero eigenvalue since the constant term $a_{4}$ of the reduced characteristic is zero. A corresponding left eigenvector, $v=\left[v_{1} v_{2} \cdots v_{6}\right]$, associated with the zero eigenvalue is given by

$$
\begin{aligned}
& v_{1}=v_{5}=0 \\
& v_{2}=\frac{K \mu_{e}}{\beta_{e}^{*}} v_{6} \\
& v_{3}=\frac{(\lambda+\mu) v_{2}-(1-\epsilon) \lambda v_{4}}{\epsilon \lambda} \\
& v_{4}=\frac{\beta_{1} v_{2}+\mu_{e} v_{6}}{\xi} \\
& v_{6}=v_{6} \neq 0
\end{aligned}
$$

and a corresponding right eigenvector, $w=\left[w_{1} w_{2} \cdots w_{6}\right]^{T}$, associated with the zero eigenvalue is given by

$$
\begin{aligned}
& w_{1}=-\frac{\lambda+\mu}{\mu} w_{2} \\
& w_{2}=w_{2} \neq 0 \\
& w_{3}=\frac{\epsilon \lambda}{\eta} w_{2} \\
& w_{4}=\frac{(1-\epsilon) \lambda}{\xi} w_{2} \\
& w_{5}=\frac{\gamma_{2} w_{3}+\gamma_{1} w_{4}}{\mu} \\
& w_{6}=\alpha w_{3}+w_{4}
\end{aligned}
$$


Now, taking the free parameters $v_{6}$ and $w_{2}$ to be positive and calculating the values of $a$ and $b$ as defined in Theorem 4.1 [5], we get

$$
\begin{aligned}
a & =-\frac{\lambda+\mu}{\mu}\left[\beta_{1} w_{4}+\beta_{2} w_{3}+\beta_{e}^{*} w_{6}\left(\frac{1}{K}+\frac{\mu C}{(\lambda+\mu) K^{2}}\right)\right] w_{2} v_{2}<0 \\
b & =\frac{w_{6} v_{2}}{K}>0
\end{aligned}
$$

Hence, using the above mentioned theorem, the direction of the bifurcation is forward, i.e. the unstable endemic equilibrium became locally asymptotically stable when $R_{0}$ crosses unity, i.e. $R_{0}>1\left(\tilde{R}_{0}>1\right)$, which concludes the proof. This result is confirmed by sketching the bifurcation diagram, taking $\beta_{e}$ to be a bifurcation parameter, as illustrated in Figure 1 .

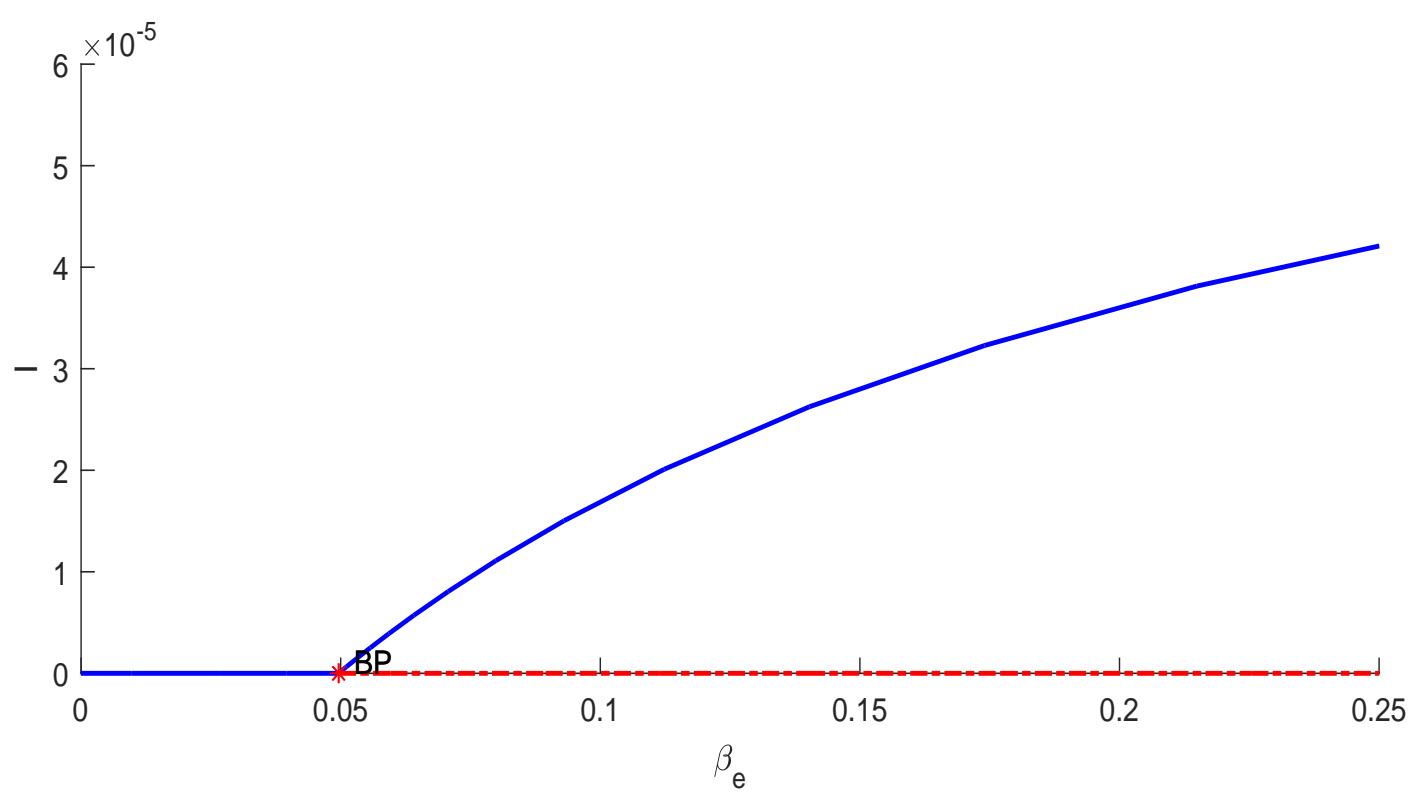

Figure 1: Bifurcation diagram with a bifurcation parameter $\beta_{e}$.

\section{Numerical Simulation}

In this section, we present some numerical simulations to demonstrate the effect of the model parameters related to the environmental transmission in the disease transmission dynamics. The parameter values are taken from [22] as listed in Table 2. Figure 2a illustrates the effect of the contact rate with contaminated environment, $\beta_{e}$. It shows that with fixed human to human transmission rates, reducing $\beta_{e}$ has the effect of not only reducing the maximum infected humans, but also delaying the time it takes to reach this maximum. However, this effect 
will be upto a certain limit since the disease transmission is dominated by human to human transmission as it can be seen for the case when $\beta_{e}=0$. The effect of human to human transmission represented by the parameter $\beta_{1}$ and $\beta_{2}$ is illustrated in Figure 2b. It can be clearly seen that the effect of environmental transmission is very low in the absence of human to human transmission. As the human to human transmission rates increase, the maximum infection increase and the time to reach this maximum is reduced. The effect of the relative shedding rate of asymptomatic, $\alpha$, is illustrated in Figure 3 . For low portion of asymptomatic humans, $\epsilon=0.13166$, there is almost no effect as expected. However, if the portion of asymptomatic is increased, $\epsilon=0.5$, a small effect of decreasing $\alpha$ is observed, which is mainly to slightly reduce the maximum infected humans as illustrated in Figure $3 \mathrm{~b}$. The effect of the relative minimum concentration of virus at environment capable of ensuring $50 \%$ chance of contracting the disease , $K$, is illustrated in Figure 4, which clearly show that the less concentration required for contracting the disease, the more infections we have and the less time it takes to reach the maximum infected human. Finally, Figure 5 shows that the initial concentration of virus at contaminated environment is enhancing the effect of the environmental transmission by increasing the maximum number of infected humans and reducing the time it takes to reach this maximum. This effect is illustrated with and without human to human transmission in Figures $5 \mathrm{a}$ and 5b, respectively. 


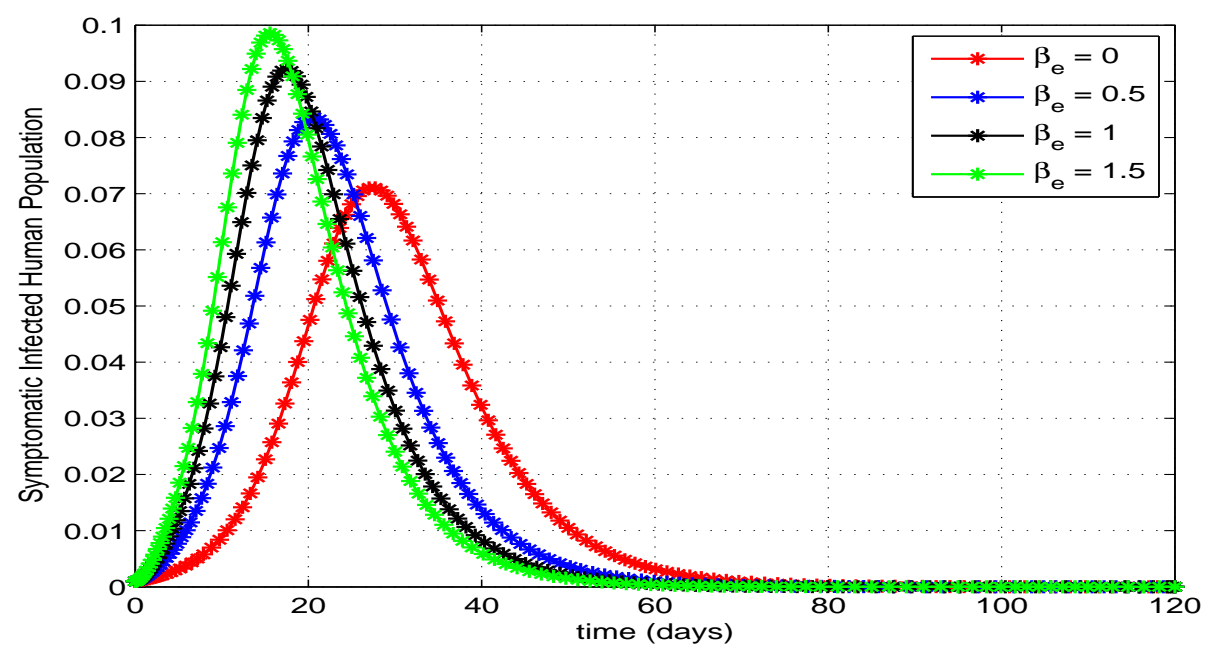

(a) Effect of the contact rate with environment $\beta_{e}$

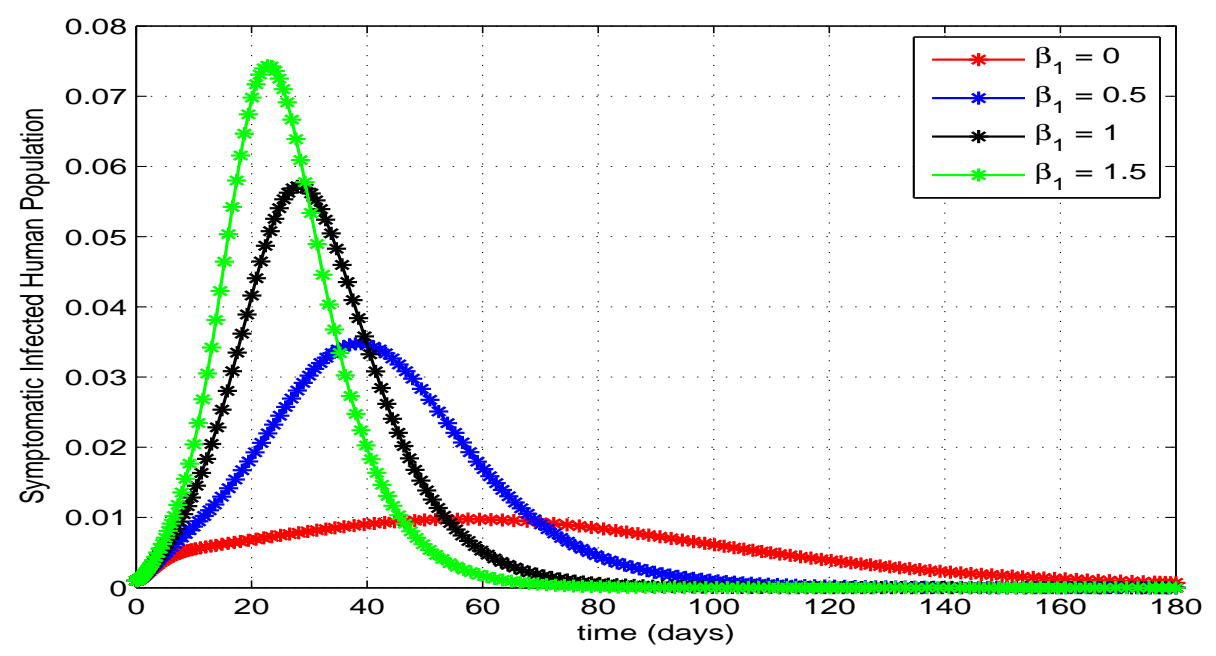

(b) Effect of human to human transmission rates with $\beta_{2}=0.45 \beta_{1}$

Figure 2: Effect of environmental and human to human transmissions 


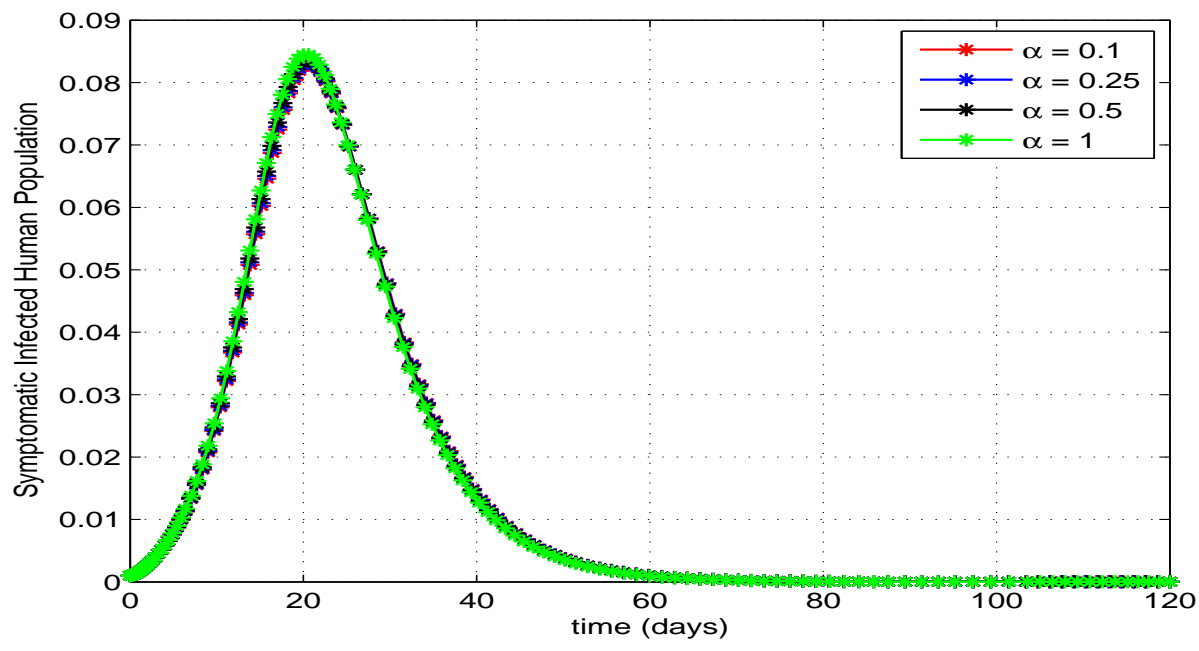

(a) Effect of relative shedding rate with $\epsilon=0.13166$

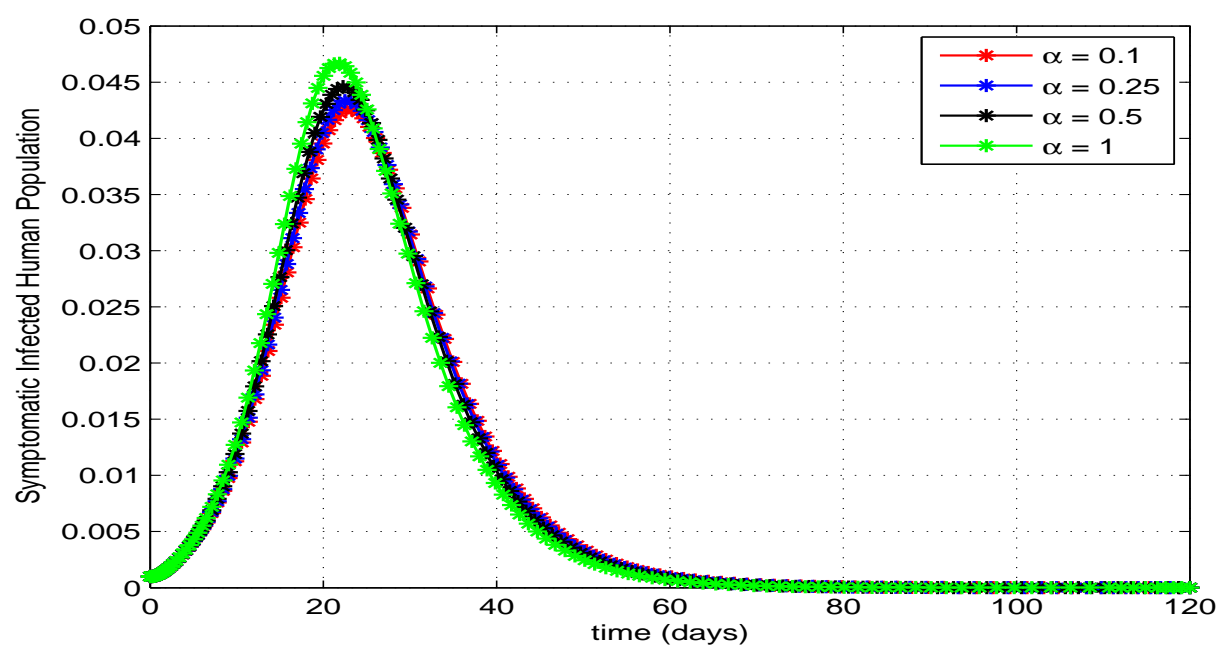

(b) Effect of relative shedding rate with $\epsilon=0.5$

Figure 3: Effect of relative shedding rate of asymptomatic 


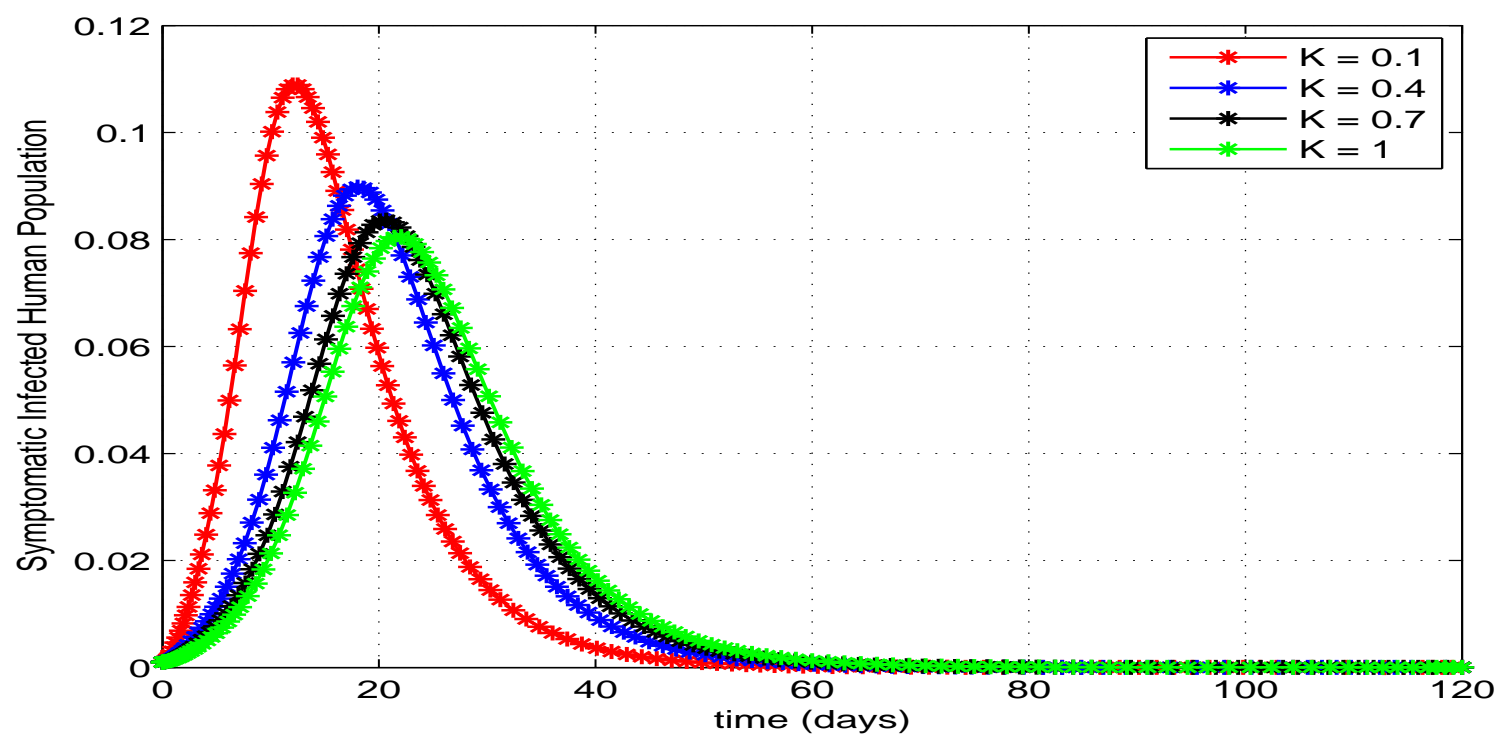

Figure 4: Effect of the virus relative concentration $K$. 


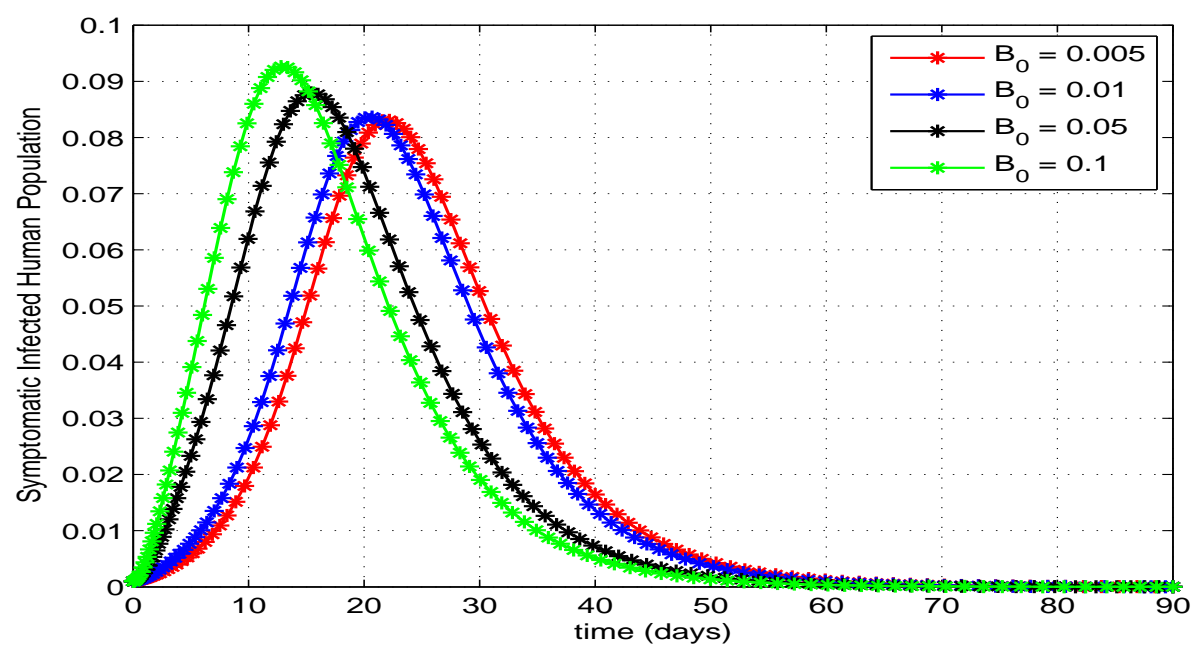

(a) Effect of initial virus concentration with $\beta_{1}=1.8457$

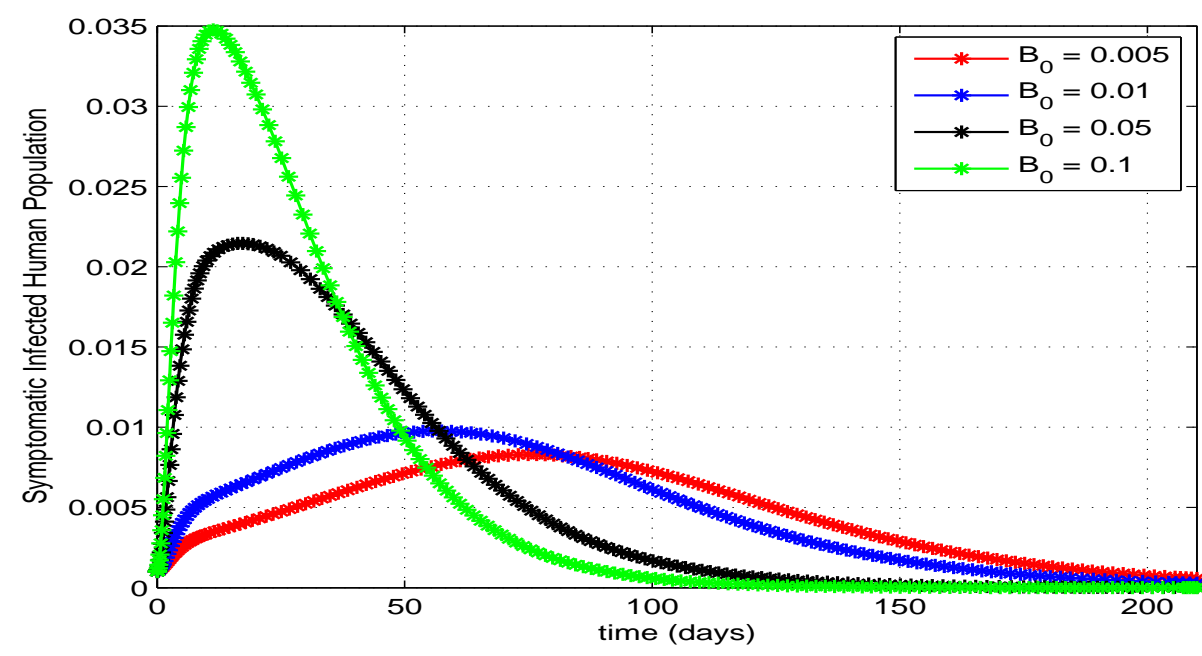

(b) Effect of initial virus concentration with $\beta_{1}=0$

Figure 5: Effect of initial virus concentration in the contaminated environment

\section{Conclusion}

In this paper, we have investigated the role of environmental transmission in COVID-19 transmission dynamics. We have proposed a mathematical model in which the environmental transmission term is represented by the expression $\frac{\beta_{e} B S}{k+B}$, where $\beta_{e}$ is the contact rate with the contaminated environment and $\frac{B}{k+B}$ is the probability of catching the disease. The basic 
reproduction number $R_{0}$ was calculated using the next generation model. Its expression includes two terms $R_{h h}$ and $R_{h e}$ corresponding to human to human and environment to human transmissions, respectively. The values of these two terms were calculated using estimated parameters from two datasets. The first dataset was from December 7, 2019 to January 1, 2020 and the second one was from January 22 to February 21, 2020. The corresponding values of $R_{h h}$ were found to be 3.25 and 3.67, respectively, which are not far from each other. However, there is a notable difference between the corresponding values of $R_{h e}$, which were estimated to 2.77 and 1.44, respectively. This difference was attributed to the closure of the Huanan Seafood Market, which took place on January 1, 2020 and hence it is expected that the environmental transmission to be less after the closure of the market. Another form for the basic reproduction number was also derived, which has a term with a square root indicating that human to human transmission via environment takes place in two steps. Using this form, the estimated values of the basic reproduction number was found to be 3.95 and 4.03 corresponding to the above mentioned datasets, respectively. Comparing these values with the corresponding values of $R_{h h}$, one would conclude that the major contribution towards the basic reproduction number is coming from human to human transmission. Sensitivity analysis of the reproduction number to the corresponding model parameters has been carried out and the results showed that the transmission rate of COVID-19 from symptomatic humans and the recovery rate of symptomatic humans are the most influential parameters in the disease transmission dynamics that the parameters related to environmental transmission may play less of a role in the diseases transmission. Existence and stability analysis of equilibrium points were presented, confirming that the disease free equilibrium is stable whenever $R_{0}<1$ and the endemic equilibrium is stable whenever $R_{0}>1$, which is true for the two proposed forms of $R_{0}$. Finally, some numerical simulations to demonstrate the effect of the parameters related to the environmental transmission have been presented. It was confirmed that COVID-19 transmission dynamics is dominated by human to human transmission with some role played by environmental transmission. In particular, reducing environmental transmission has the effect of reducing the maximum infection and delaying the time to reach this maximum upto a certain limit. This can be achieved by strictly adhering to environmental and hand hygiene. 


\section{References}

[1] WHO - WHO Director-General's opening remarks at the media briefing on COVID-19 - 11 March 2020, url = https://www.who.int/dg/speeches/detail/who-director-general-sopening-remarks-at-the-media-briefing-on-covid-19-11-march-2020, year = 2020.

[2] WHO - Naming the coronavirus disease (COVID-19) and the virus that causes it, 2020.

[3] WHO - Novel Coronavirus China, Disease outbreak news : Update 12 January 2020, 2020 .

[4] S. Al-Shanfari, I.M. Elmojtaba, and N. Al-Salti, The role of houseflies in cholera transmission, Communications in Mathematical Biology and Neuroscience 2019 (2019).

[5] C. Castillo-chavez and B. Song, Dynamical models of tuberculosis and their applications, Mathematical Biosciences and Engineering 1 (2004), no. 2, 361404.

[6] Feng Z. Castillo-Chavez, C. and W. Huang, On the computation of ro and its role on global stability, In: Castillo-Chavez, P.C., Blower, S., Driessche, P., Kirschner, D. and Yakubu, A.-A., Eds., Mathematical Approaches for Emerging and Reemerging Infectious Diseases: An Introduction, Springer, Berlin 1 (2002), 229.

[7] Tian Mu Chen, Jia Rui, Qiu Peng Wang, Ze Yu Zhao, Jing An Cui, and Ling Yin, A mathematical model for simulating the phase-based transmissibility of a novel coronavirus, Infect. Dis. Poverty 9 (2020), no. 1, 24.

[8] O. Diekmann, J. A.P. Heesterbeek, and J. A.J. Metz, On the definition and the computation of the basic reproduction ratio $R 0$ in models for infectious diseases in heterogeneous populations, Journal of Mathematical Biology 28 (1990), no. 4, 365-382.

[9] Paul Digard, Is the minimum infective dose of covid-19 small?, 2020.

[10] S. F. Dowell, J. M. Simmerman, D. D. Erdman, J.-S. J. Wu, A. Chaovavanich, M. Javadi, J.-Y. Yang, L. J. Anderson, S. Tong, and M. S. Ho, Severe Acute Respiratory Syndrome Coronavirus on Hospital Surfaces, Clin. Infect. Dis. 39 (2004), no. 5, 652-657.

[11] Avner Friedman and Abdul Aziz Yakubu, Anthrax epizootic and migration: Persistence or extinction, Mathematical Biosciences 241 (2013), no. 1, 137-144.

[12] D. M. Hamby, A review of techniques for parameter sensitivity analysis of environmental models, Environmental Monitoring and Assessment 32 (1994), 135-154,. 
[13] John S Ji, Origins of MERS-CoV, and lessons for 2019-nCoV, Lancet Planet. Heal. (2020).

[14] Günter Kampf, Daniel Todt, Stephanie Pfaender, and Eike Steinmann, Persistence of coronaviruses on inanimate surfaces and its inactivation with biocidal agents, J. Hosp. Infect. 104 (2020), 246-251.

[15] Adam J. Kucharski, Timothy W. Russell, Charlie Diamond, Yang Liu, John Edmunds, Sebastian Funk, Rosalind M. Eggo, Fiona Sun, Mark Jit, James D. Munday, Nicholas Davies, Amy Gimma, Kevin van Zandvoort, Hamish Gibbs, Joel Hellewell, Christopher I. Jarvis, Sam Clifford, Billy J. Quilty, Nikos I. Bosse, Sam Abbott, Petra Klepac, and Stefan Flasche, Early dynamics of transmission and control of COVID-19: a mathematical modelling study, Lancet Infect. Dis. 3099 (2020), no. 20, 1-7.

[16] Qianying Lin, Shi Zhao, Daozhou Gao, Yijun Lou, Shu Yang, Salihu S. Musa, Maggie H. Wang, Yongli Cai, Weiming Wang, Lin Yang, and Daihai He, A conceptual model for the coronavirus disease 2019 (COVID-19) outbreak in Wuhan, China with individual reaction and governmental action, Int. J. Infect. Dis. 93 (2020), 211-216.

[17] Sean Wei Xiang Ong, Yian Kim Tan, Po Ying Chia, Tau Hong Lee, Oon Tek Ng, Michelle $\mathrm{Su}$ Yen Wong, and Kalisvar Marimuthu, Air, Surface Environmental, and Personal Protective Equipment Contamination by Severe Acute Respiratory Syndrome Coronavirus 2 (SARS-CoV-2) from a Symptomatic Patient, JAMA - J. Am. Med. Assoc. (2020), 3-5.

[18] J. A. Otter, C. Donskey, S. Yezli, S. Douthwaite, S. D. Goldenberg, and D. J. Weber, Transmission of SARS and MERS coronaviruses and influenza virus in healthcare settings: The possible role of dry surface contamination, 92 (2016), no. 3, 235-250.

[19] Julien Riou and Christian L. Althaus, Pattern of early human-to-human transmission of Wuhan 2019 novel coronavirus (2019-nCoV), December 2019 to January 2020, Eurosurveillance 25 (2020), no. 4, 1-5.

[20] Hussin A. Rothan and Siddappa N. Byrareddy, The epidemiology and pathogenesis of coronavirus disease (COVID-19) outbreak, J. Autoimmun. 109 (2020), 102433.

[21] C. M. Saad-Roy, P. van den Driessche, and Abdul Aziz Yakubu, A mathematical model of anthrax transmission in animal populations, Bulletin of Mathematical Biology 79 (2017), no. 2, 303-324.

[22] Sk Shahid Nadim, Indrajit Ghosh, and Joydev Chattopadhyay, Short-term predictions and prevention strategies for COVID-2019: A model based study, arXiv (2020). 
[23] Biao Tang, Nicola Luigi Bragazzi, Qian Li, Sanyi Tang, Yanni Xiao, and Jianhong Wu, An updated estimation of the risk of transmission of the novel coronavirus (2019-nCov), Infect. Dis. Model. 5 (2020), 248-255.

[24] C Torres Codeco, Endemic and epidemic dynamics of cholera: The role of the aquatic reservoir, BMC Infect. Dis. 1 (2001), no. 1.

[25] Pauline van den Driessche, Reproduction numbers of infectious disease models, Infectious Disease Modelling 2 (2017), no. 3, 288-303.

[26] Joseph T. Wu, Kathy Leung, and Gabriel M. Leung, Nowcasting and forecasting the potential domestic and international spread of the 2019-nCoV outbreak originating in Wuhan, China: a modelling study, Lancet 6736 (2020), no. 20.

[27] Hyun Mo Yang, The basic reproduction number obtained from jacobian and next generation matrices - a case study of dengue transmission modelling, BioSystems 126 (2014), 52-75.

[28] Shi Zhao, Qianyin Lin, Jinjun Ran, Salihu S. Musa, Guangpu Yang, Weiming Wang, Yijun Lou, Daozhou Gao, Lin Yang, Daihai He, and Maggie H. Wang, Preliminary estimation of the basic reproduction number of novel coronavirus (2019-nCoV) in China, from 2019 to 2020: A data-driven analysis in the early phase of the outbreak, Int. J. Infect. Dis. 92 (2020), 214-217.

[29] Tao Zhou, Quanhui Liu, Zimo Yang, Jingyi Liao, Kexin Yang, Wei Bai, Xin Lu, and Wei Zhang, Preliminary prediction of the basic reproduction number of the Wuhan novel coronavirus 2019-nCoV, J. Evid. Based. Med. 13 (2020), no. 1, 3-7.

\section{Declarations:}

Competing interests: The authors declare no competing interests. 
Figures

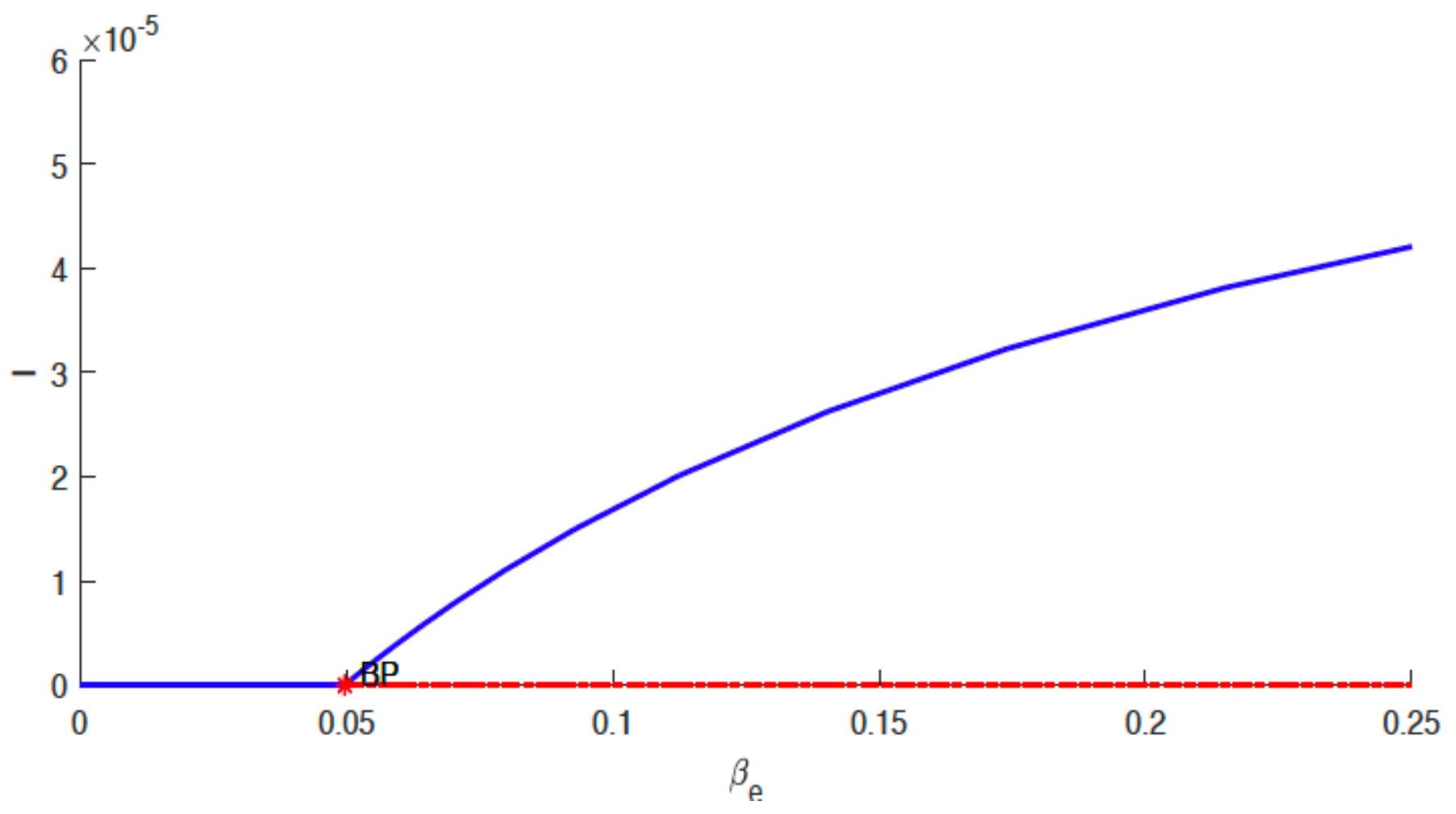

Figure 1

Bifurcation diagram with a bifurcation parameter $\beta e$. 


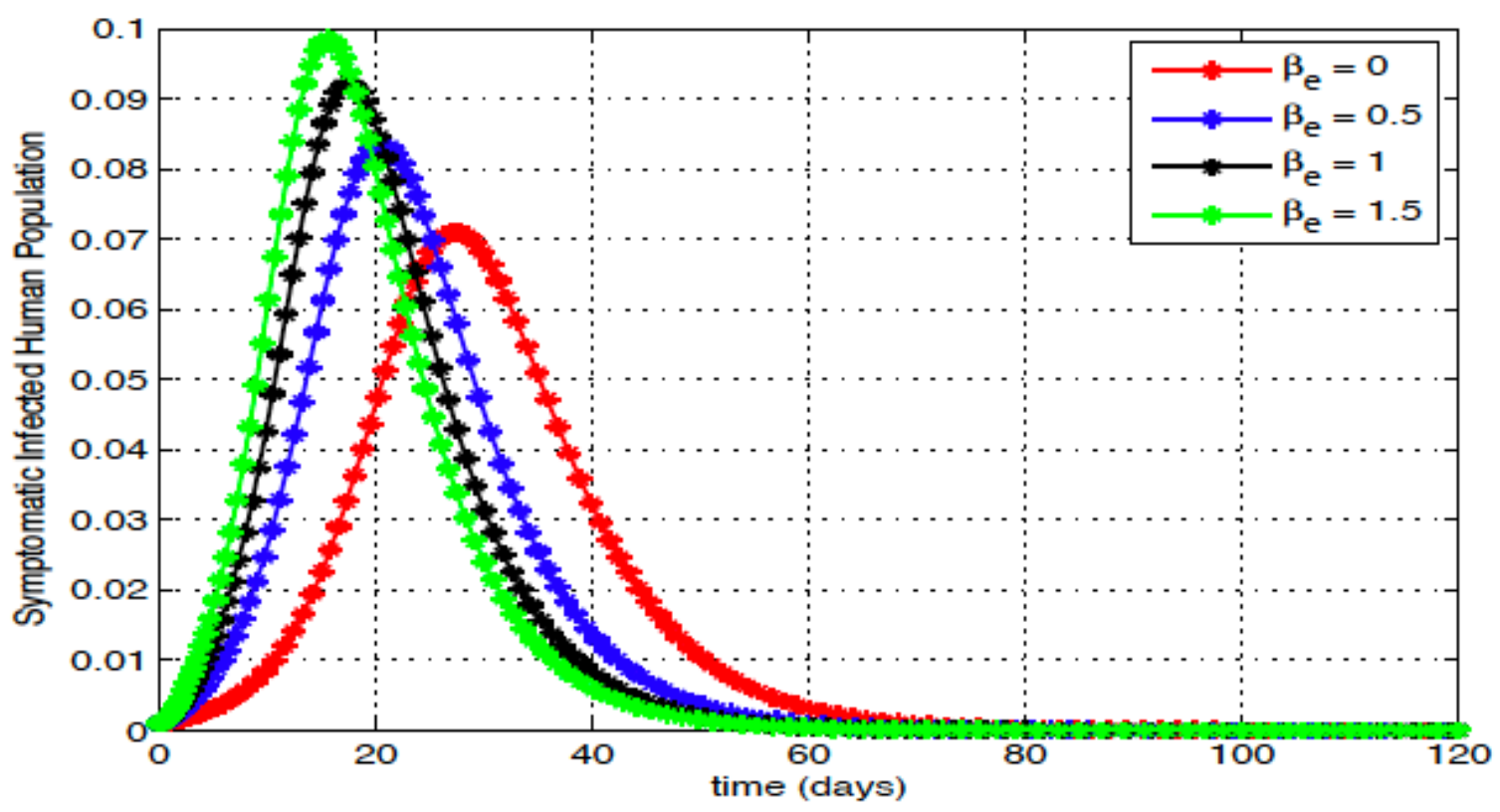

(a) Effect of the contact rate with environment $\beta_{e}$

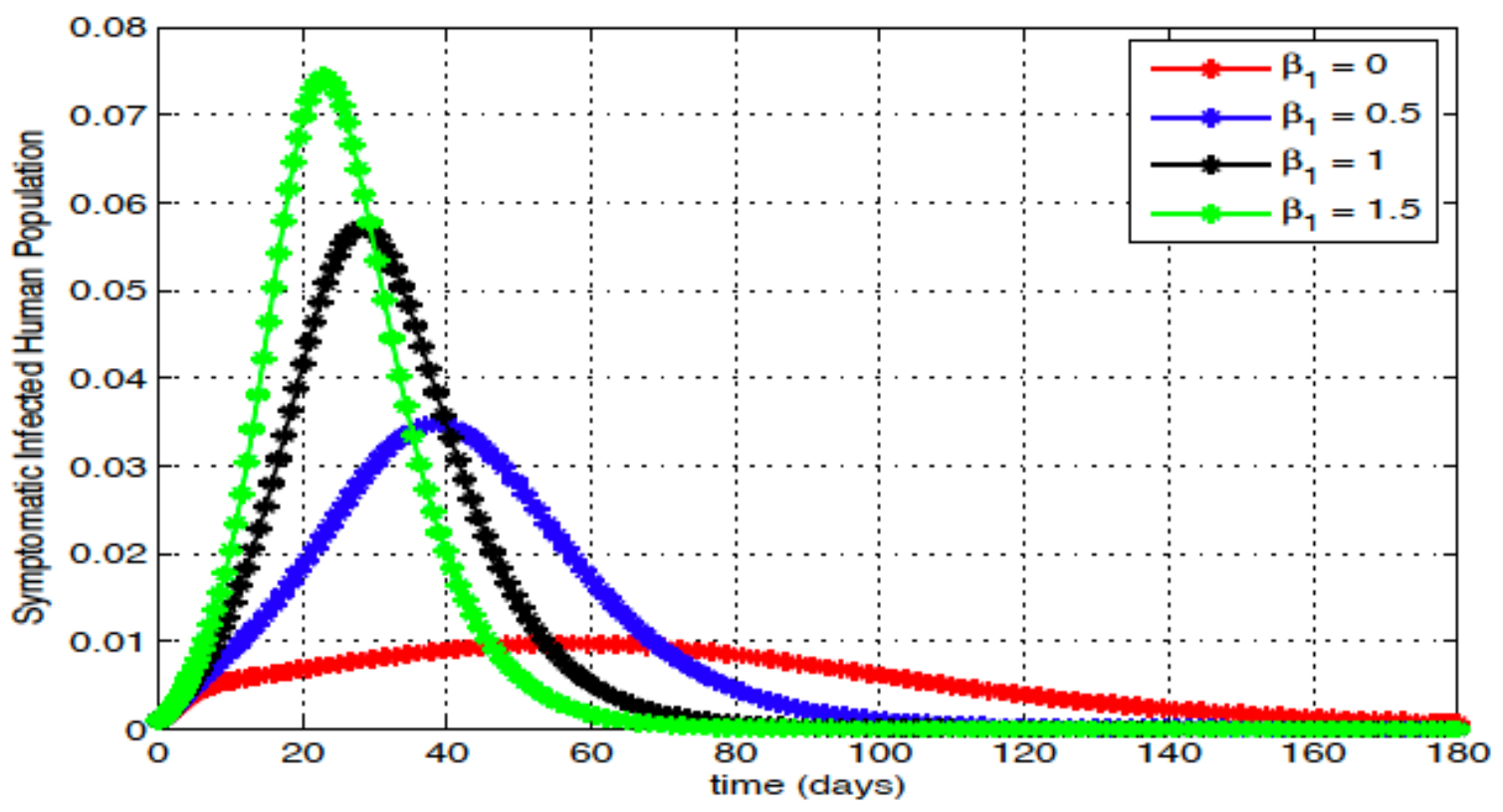

(b) Effect of human to human transmission rates with $\beta_{2}=0.45 \beta_{1}$

Figure 2

Effect of environmental and human to human transmissions 


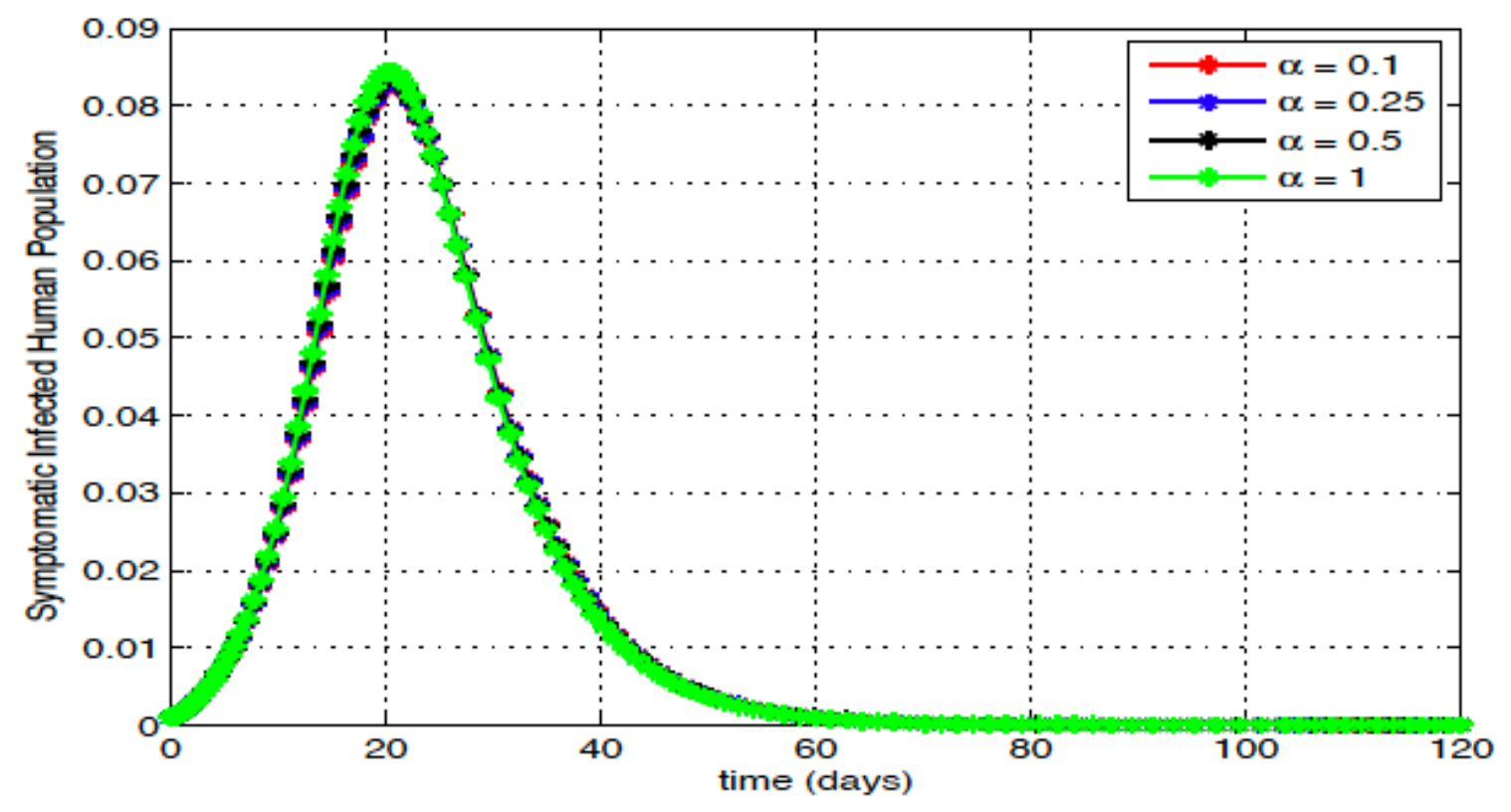

(a) Effect of relative shedding rate with $\epsilon=0.13166$

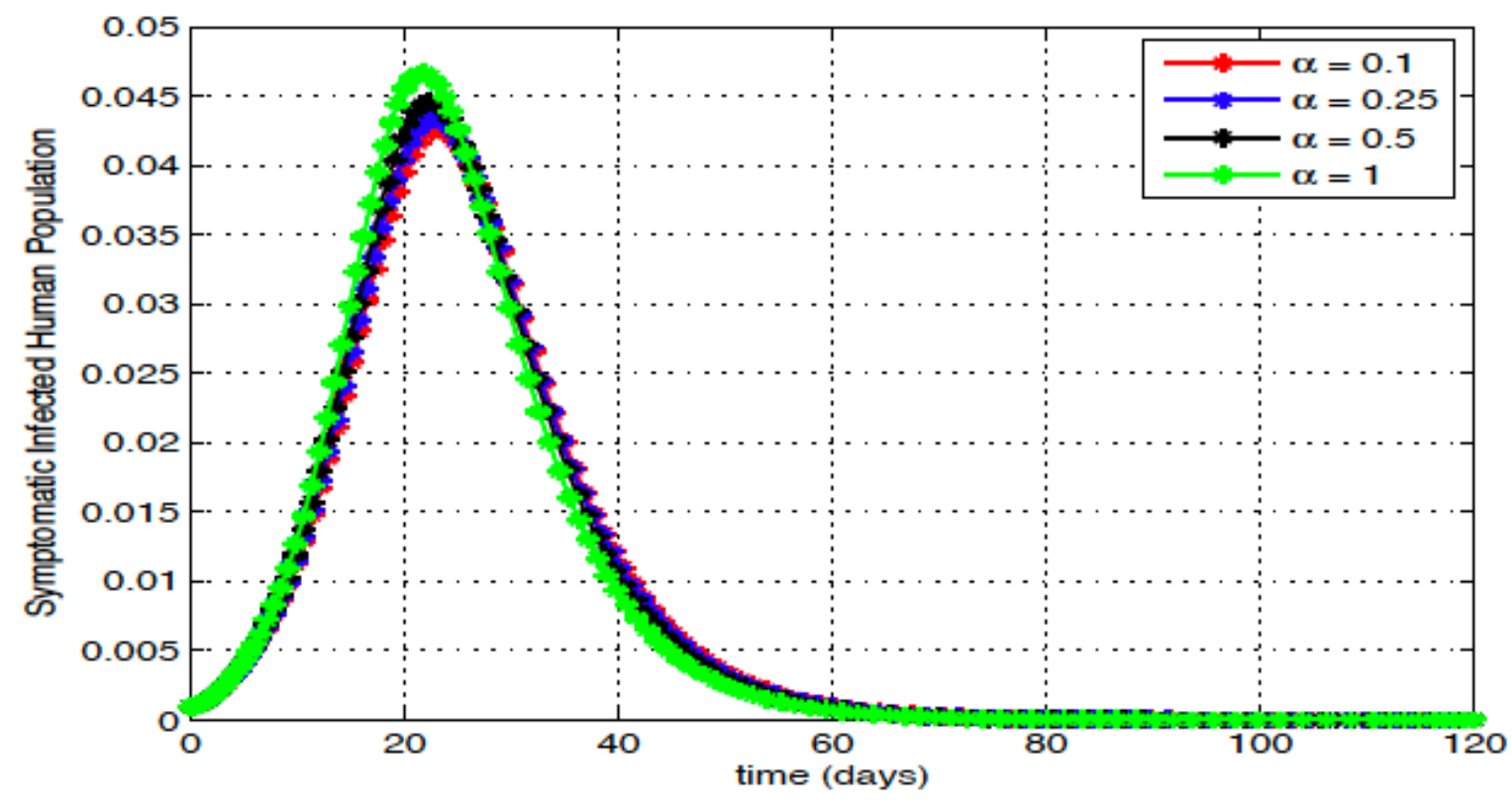

(b) Effect of relative shedding rate with $\epsilon=0.5$

Figure 3

Effect of relative shedding rate of asymptomatic 


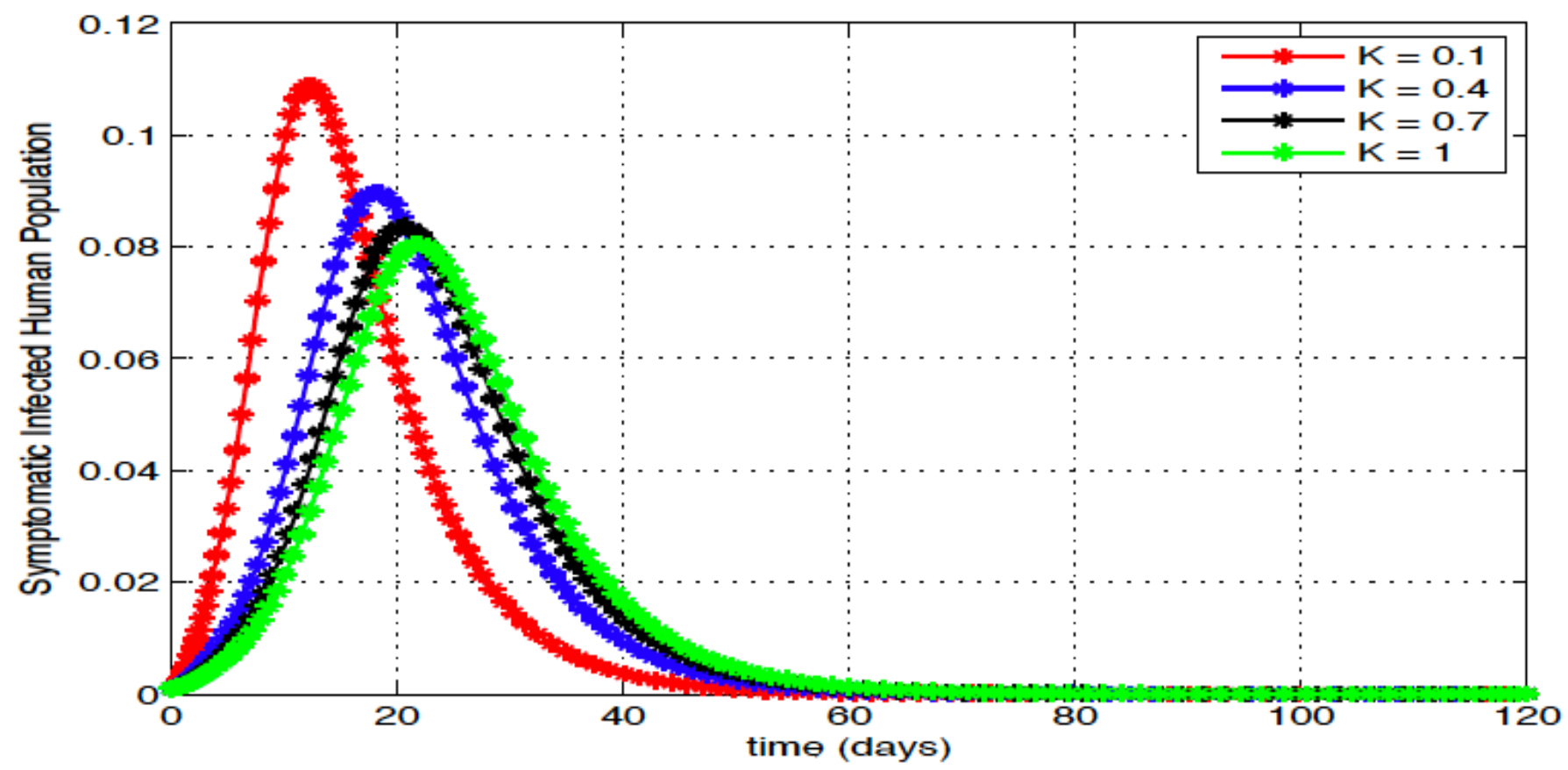

Figure 4

Effect of the virus relative concentration $\mathrm{K}$. 


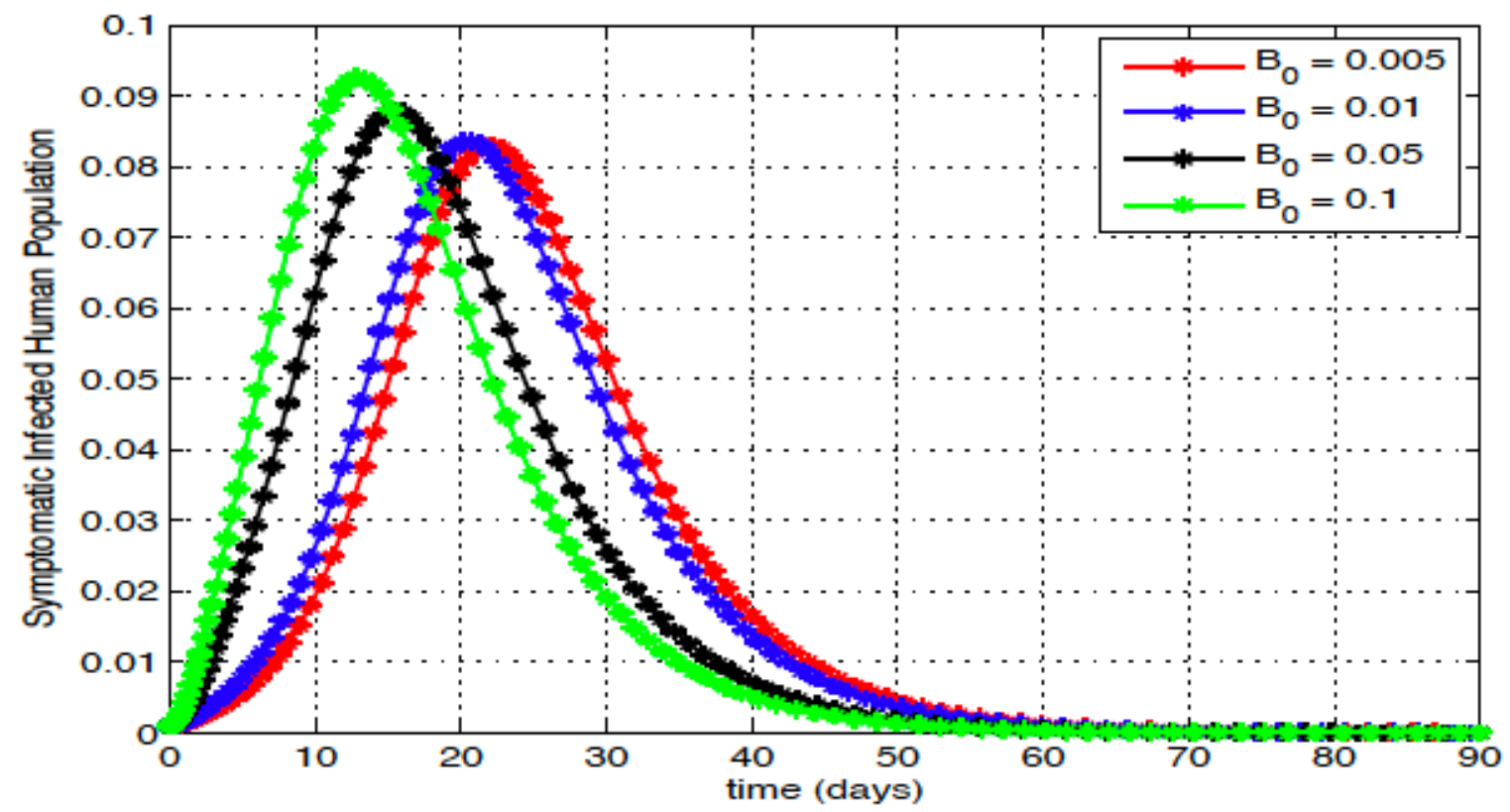

(a) Effect of initial virus concentration with $\beta_{1}=1.8457$

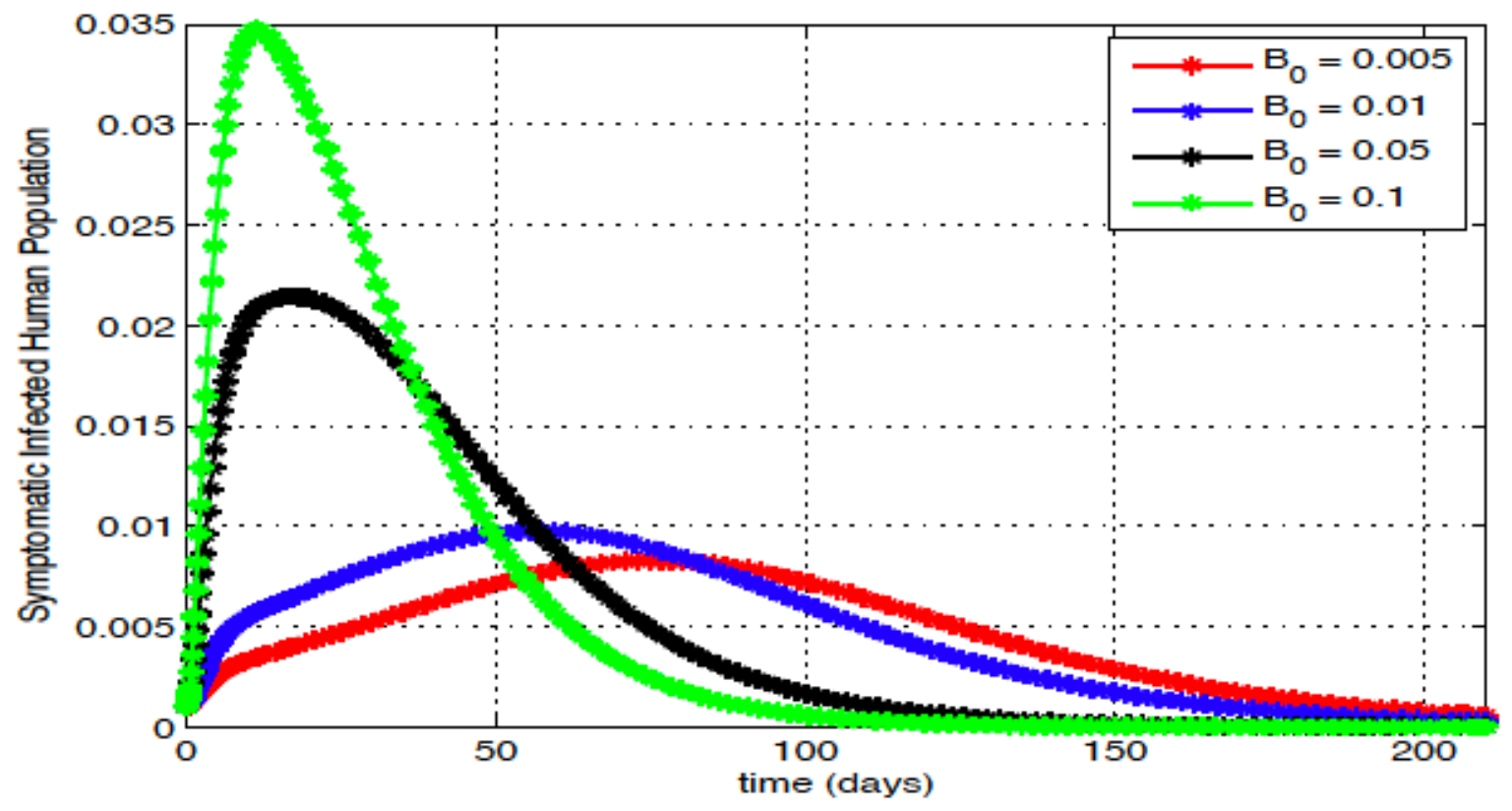

(b) Effect of initial virus concentration with $\beta_{1}=0$

\section{Figure 5}

Effect of initial virus concentration in the contaminated environment 